\title{
Computational Expenditure Reduction in Pseudo-Gradient Image Parameter Estimation
}

\author{
Alexandr Tashlinskii \\ Ulyanovsk State Technical University, 32 Severnyi Venetz Str., 432027 Ulyanovsk \\ Russia \\ tag@ulstu.ru
}

\begin{abstract}
An approach enabling to reduce computational expenses at pseudogradient estimation of image parameters based on control of goal function local sample volume is proposed. Local sample volume variation during the process of parameter estimation occurs automatically in correspondence with a preassigned criterion, for instance, sample correlation coefficient. It is shown that for the problems of image mutual spatial deformation parameter estimation, computational expenses can be reduced several times as many.
\end{abstract}

\section{Introduction}

For remote exploration of the Earth, in medicine, geology and navigation information extraction systems with embedded spatial apertures of signal sensors are becoming more and more popular. Such systems contain initial data in the form of dynamic arrays with proper feature which is in their space-time correlation and due to this they can be represented in the form of multidimensional images(MI).

When developing algorithmic software of MI processing it is necessary to take into account the dynamics of the scene to be observed, spatial movements of the signal sensors and imperfection of their construction. The influence of the mentioned factors can be described through mathematical models of space-time deformations of multidimensional grids with assigned image. The estimation of the varying parameters of spatial deformations (SD) is required at automated search of a fragment on image, navigational tracking of the mobile object course in the conditions of limited visibility, combination of multidomain images at remote explorations of the Earth, in medical explorations and other problems. A great number of scientific publications are devoted to the aspects of image space-time deformation parameter estimation. In the present work ways of computational expense reduction when using pseudogradient procedures to solve this problem are considered.

Let us assume that the model of MI SD is defined with accuracy of parameters vector $\bar{\alpha}$ and estimation quality criterion $\bar{\alpha}$ is formulated in terms of some functional $J(\bar{\alpha})$ minimization showing expected losses. However it is impossible to find the optimal parameters $\bar{\alpha}^{*}$ in the appointed sense in view of incompleteness of MI description. In this case the parameters $\bar{\alpha}$ can be estimated on the 
basis of MI realization analysis by means of some adaptation procedure which minimizes $J(\bar{\alpha})=J(\bar{\alpha}, Z)$ for a given realization. However it is reasonable to avoid this intermediate stage of exploration and determine $\bar{\alpha}$ directly on values $J(\bar{\alpha})=J(\bar{\alpha}, Z)[1]$ :

$$
\bar{\alpha}_{t}=\bar{\alpha}_{t-1}-\boldsymbol{\Lambda}_{t} \nabla J\left(\bar{\alpha}_{t-1}, Z\right)
$$

where $\bar{\alpha}_{t}$ is next after $\bar{\alpha}_{t-1}$ approximation of minimum point; $\boldsymbol{\Lambda}_{t}$ is positively defined matrix determining step value; $\nabla J\left(\bar{\alpha}_{t-1}, Z\right)$ is gradient of the functional $J\left(\bar{\alpha}_{t-1}, Z\right)$. The application of the procedure (1) in image processing is prevented by the necessity of multiple cumbersome calculations of $\nabla J\left(\bar{\alpha}_{t-1}, Z\right)$. It is possible to essentially decrease the volume of calculations if instead of $\nabla J\left(\bar{\alpha}_{t-1}, Z\right)$ we use its contraction $\nabla Q_{t}=\nabla J\left(\bar{\alpha}_{t-1}, Z_{t}\right)$ at some part $Z_{t}$ of the realization choosing, for example, in the capacity of $Z_{t}$ a sliding window. The analysis of the approaches [2-5] to the synthesis of large MI SD estimation procedures in real time showed that the algorithms satisfying the requirements of simplicity, fast convergence and capacity to work in various real situations are reasonable to seek in the class of recurrent non-identification adaptive algorithms and the most representative group of these algorithms constitutes pseudogradient algorithms (PGA). The concept of pseudogradient (PG), which was the basis for development of a unified approach to the analysis and synthesis of various procedures of functionals stochastic minimization, was introduced by B.T.Polyak and Y.Z.Tzypkin in work [6] The class of PGA is very wide and includes algorithms of stochastic approximation, random search and many others.

In PGA the following procedure [1] is used

$$
\hat{\bar{\alpha}}_{t}=\hat{\bar{\alpha}}_{t-1}-\boldsymbol{\Lambda}_{t} \bar{\beta}_{t}
$$

where $\bar{\alpha}$ is vector of the parameters to be estimated; $\boldsymbol{\Lambda}_{t}$ is positively defined matrix usually called amplification matrix; $\bar{\beta}_{t}$ is some random direction in the parameter space depending on the values $\hat{\bar{\alpha}}_{t-1}$ and on step number $t$; $\hat{\bar{\alpha}}_{0}$ is initial approximation of the parameters vector. The direction $\bar{\beta}_{t}$ will be PG if the condition $\left[\nabla J\left(\hat{\bar{\alpha}}_{t-1}\right)\right]^{T} M\left\{\bar{\beta}_{t}\right\} \geq 0$ is satisfied, i.e. if the vector $\bar{\beta}_{t}$ makes on the average a sharp angle with an exact value of functional gradient. The algorithm [2] will be considered to be pseudogradient, if $\bar{\beta}_{t}$ is PG at each its step. In this case the steps in (2) are performed on average in the direction of reduction of $J(\hat{\bar{\alpha}})$ and sequence $\hat{\bar{\alpha}}_{1}, \hat{\bar{\alpha}}_{2}, \ldots$ will converge to the minimum point $\hat{\alpha}^{*}$ when satisfying relatively weak conditions [7]).

We should mention that in the algorithm (2) a possibility of calculation of $J\left(\hat{\bar{\alpha}}_{t-1}, Z_{t}\right)$ or $\nabla J\left(\hat{\bar{\alpha}}_{t-1}, Z_{t}\right)$ is not assumed, i.e. $J(\hat{\bar{\alpha}})$ can be non-observable. It is necessary to satisfy only the condition of pseudogradientness. In particular, in the capacity of $J\left(\bar{\beta}_{t}\right)$ we can choose a noisy value of some other functional $J_{d}(\hat{\alpha})$ which has the same point of minimum $\bar{\alpha}^{*}$. Admissibility of the dependence $\bar{\beta}_{t}$ on the preceding values $\hat{\alpha}_{t}$ enables a possibility of PGA application for MI processing in the order of some sweep. In this case $\bar{\beta}_{t}$ can depend on the values $\hat{\alpha}_{t}$ calculated in advance in the preceding rows of the image. 
When synthesizing PGA for an assigned goal function (GF) it is necessary to derive some easily calculated PG. Below computational expense reduction ways are considered when designing $\mathrm{PG}$.

\section{Pseudogradient Kind Selection}

When synthesising PGA (2) the most important moment is in finding the performance index PG $J(\hat{\alpha}, Z)$ or its contraction $J\left(\bar{\alpha}, Z_{t}\right)$. In the work [8] it is shown that if two frames $\mathbf{z}^{(1)}=\left\{z_{\bar{j}}^{(1)}=x_{\bar{j}}+\theta_{\bar{j}}^{(1)}\right\}$ and $\mathbf{z}^{(2)}=\left\{z_{\bar{j}}^{(2)}=x(\bar{j}, \bar{\alpha})+\theta_{\bar{j}}^{(2)}\right\}$ of MI, defined on grid of samples $\Omega:\left\{\bar{j}=\left(j_{1}, j_{2}, \ldots, j_{n}\right)\right\}$ constitute additive mixture of informational $\left\{x_{\bar{j}}\right\}$ and white $\left\{\theta_{\bar{j}}\right\}$ random fields then when minimising GF PG can be obtained at the expense simplification of the gradient

$$
\nabla J(\bar{\alpha}, Z)=\sum_{\bar{j}, \bar{l} \in \Omega} \frac{\partial x(\bar{j}, \bar{\alpha})}{\partial \bar{\alpha}} V_{Z \bar{j} \bar{l}}^{-1}\left(x_{\bar{l}}(\bar{l}, \bar{\alpha})-z_{\bar{l}}^{(2)}\right)
$$

and when GF maximising is gradient

$$
\nabla J(\bar{\alpha}, Z)=-\sum_{\bar{j}, \bar{l} \in \Omega} \frac{\partial x(\bar{j}, \bar{\alpha})}{\partial \bar{\alpha}} V_{Z \bar{j} \bar{l}}^{-1} z_{\bar{l}}^{(2)}
$$

where $V_{Z \bar{j} \bar{l}}$ is covariance matrix of the conditional distribution $w\left(\left\{z_{\bar{j}}^{(2)}\right\} /\right.$ $\left.\left\{z_{\bar{j}}^{(1)}, \bar{\alpha}\right\}\right) ; x(\bar{j}, \bar{\alpha})$ is deformed frame samples prediction. Reduction of operations number in (3) and (4) can be obtained using the contraction of $\nabla Q_{t}=$ $\nabla J\left(\hat{\bar{\alpha}}_{t-1}, Z_{t}\right)$, where $Z_{t}=\left\{z_{\bar{j}, t}^{(2)}, x\left(\bar{j}, \hat{\bar{\alpha}}_{t}\right)\right\}$ local sample of GF $Q$ at $t$-th iteration; $z_{\bar{j}, t}^{(2)} \in \mathbf{z}^{(2)}, \bar{j}_{t} \in \Omega_{t} \in \Omega$, and substituting the prediction $x(\bar{j}, \bar{\alpha})$ of deformed frame values by a simpler estimate. This estimate can be obtained, for example, on the basis of interpolation with the estimates $\bar{\alpha}$ used in the capacity of its parameters at next iteration due and obtained at a preceding iteration. Then the local sample of GF at the $t$-th iteration will be

$$
Z_{t}=\left\{z_{\bar{j} t}^{(2)}, \tilde{z}_{\bar{j} t}^{(2)}\right\} ; z_{\bar{j} t}^{(2)} \in \mathbf{z}^{(2)} ; \tilde{z}_{\bar{j} t}^{(1)}=\tilde{z}^{(1)}\left(\bar{j}_{t}, \hat{\alpha}_{t-1}\right) \in \tilde{\mathbf{z}}
$$

where $\tilde{\mathbf{z}}$ is continuous image obtained from $\mathbf{z}^{(2)}$ by means of interpolation; and the relations (3) and (4) for PG will take the form

$$
\begin{gathered}
\bar{\beta}_{t}=\nabla Q_{t}=\left.\sum_{\bar{j}_{t} \in \Omega_{t}} \frac{\partial \tilde{z}^{(1)}\left(\bar{j}_{t}, \bar{\alpha}\right)}{\partial \bar{\alpha}}\left(\tilde{z}^{(1)}\left(\bar{j}_{t}, \bar{\alpha}\right)-z_{\bar{j} t}^{(2)}\right)\right|_{\bar{\alpha}=\hat{\bar{\alpha}}_{t-1}} \\
\bar{\beta}_{t}=-\nabla Q_{t}=-\left.\sum_{\bar{j}_{t} \in \Omega_{t}} \frac{\partial \tilde{z}^{(1)}\left(\bar{j}_{t}, \bar{\alpha}\right)}{\partial \bar{\alpha}} z_{\bar{j} t}^{(2)}\right|_{\bar{\alpha}=\hat{\bar{\alpha}}_{t-1}}
\end{gathered}
$$

We should note that the formula (5) corresponds to the problem of interframe difference mean square minimisation and the formula (6) is the problem of maximization of interframe correlation sample coefficient (ICSC). 
In a number of cases in the capacity of GF PG $Q$ it is convenient to choose $\bar{\beta}_{t}=\bar{\varphi}\left(\nabla Q_{t}\right)$, where $\bar{\varphi}$ is vector function of the same dimensionality as $\nabla Q$. In particular, simple and at the same time fast converging algorithms can be obtained when selecting a sign function $\bar{\beta}_{t}=\operatorname{sgn}(\nabla Q)$ in the capacity of $\bar{\varphi}$.

\section{Algorithms with Varying Local Sample Size}

In a number of cases the problem of identification (recognition) with decisive rule based on GF values is solved together with the problem of SD estimation. In particular, when searching a fragment position on supporting image, having in relation with the supporting image in addition to SD close to linear brightness distortions, excess of some value of sample correlation coefficient between fragment and supporting image can serve as the criterion of correspondence. In this case for obtaining high confidential probability a large volume $\mu$ of GF local sample is required which can not prove itself in the process of convergence of the estimates $\bar{\alpha}$. In such situations it is reasonable to use varying $\mu$, the value of which is regulated automatically during the process of $\mathrm{PG}$ algorithm operation and is minimal for some criterion attainment at each iteration. Let us consider an example of such PGA maximizing ICSC and which showed high efficiency when solving the problem of fragment search on a supporting image. Another example is considered in work [9].

Let us choose the value $q_{t}\left(Z, \hat{\bar{\alpha}}_{t-1}, \mu_{t}\right)$ of GF estimate $Q$ as a magnitude defining $\mu_{t}$ at $t$-th iteration $q_{t}\left(Z, \hat{\bar{\alpha}}_{t-1}, \mu_{t}\right)$. Then it is expedient to use (7) in the capacity of GF PG, having complemented it with sign function for attainment of stability of parameters $\bar{\alpha}$ estimates in the condition of noise

$$
\operatorname{sgn}\left(\sum_{\bar{j}_{t} \in \Omega_{t}}\left(z_{+}^{(1)}\left(\bar{j}_{t}\right)-z_{-}^{(1)}\left(\bar{j}_{t}\right)\right)\left(z_{\bar{j} t}^{(2)}-z_{m}^{(2)}\right) /\left(\mu \hat{\sigma}_{x 1} \hat{\sigma}_{x 2}\right)\right),
$$

where $z_{m}^{(2)}=\frac{1}{\mu} \sum_{\bar{j}_{t} \in \Omega_{t}} z_{\bar{j}}^{(2)}$ is mean value of the samples $\left\{z_{\bar{j} t}^{(2)}\right\} \in Z_{t} ; z_{t( \pm)}^{(1)}=$ $\tilde{z}^{(1)}\left(\bar{j}_{t}, \alpha_{1(t-1)}, \ldots, \alpha_{i(t-1)} \pm \triangle_{\alpha_{i}}, \alpha_{m(t-1)}\right) ; \triangle_{\alpha i}>0$ is increment of the parameter $\alpha_{i}$, selected for estimation of the derivative in (6); $\hat{\sigma}_{z 1}$ and $\hat{\sigma}_{z 2}$ are estimates of the variances $\left\{z_{\bar{j} t}^{(2)}\right\}$ and $\left\{\tilde{z}_{\bar{j} t}^{(1)}\right\}$. Correspondingly if the ICSC estimate is less than $r_{c r}$, then a step in accordance with the PG value is carried out

$$
\beta_{i, t}\left(\mu_{t}\right)=\left\{\begin{aligned}
1, & q_{t+}\left(\mu_{t}\right)>q_{t-}\left(\mu_{t}\right) \\
0, & q_{t+}\left(\mu_{t}\right)=q_{t-}\left(\mu_{t}\right) ; \quad i=\overline{1, m} \\
-1, & q_{t+}\left(\mu_{t}\right)<q_{t-}\left(\mu_{t}\right)
\end{aligned}\right.
$$

where

$$
\mu_{t}=\left\{\begin{array}{rl}
\mu_{t, k}+1, & q_{t_{0}}\left(\mu_{t, k}\right) \\
\mu_{t, k}, \quad q_{t_{0}}\left(\mu_{t, k}\right) & <r_{c r} ;
\end{array} \quad \mu_{t 0}=\mu_{\min } ;\right.
$$

$q_{t-}\left(\mu_{t}\right), q_{t+}\left(\mu_{t}\right)$ and $q_{t_{0}}\left(\mu_{t}\right)$ are GF estimates respectively at $Z_{t}=$ $\left\{z_{\bar{j} t}^{(2)}, \tilde{z}_{-}^{(1)}\left(\bar{j}_{t}\right)\right\}, Z_{t}=\left\{z_{\bar{j} t}^{(2)}, \tilde{z}_{+}^{(1)}\left(\bar{j}_{t}\right)\right\}$ and $Z_{t}=\left\{z_{j t}^{(2)} \tilde{z}^{(1)}\left(\bar{j}_{t}, \hat{\bar{\alpha}}_{t-1}\right)\right\} ; k=$ 
$\overline{0,\left(k_{\max }-k_{\min }\right)} ; r_{c r}$ is ICSC value at local sample volume $\mu_{\max }$, enabling to make a decision about correspondence of a fragment to some domain of a reference image with a pre assigned validity. Thus, at first local sample volume is equal to $\mu_{\text {min }}$ at each iteration. If the ICSC estimate exceeds $r_{c r}$, then the value $\mu_{t, k}$ increases until the condition $q_{t_{0}}\left(\mu_{t, k}\right)<r_{c r}$ is satisfied, or $\mu_{t, k}$ attains the value $\mu_{\max }$.

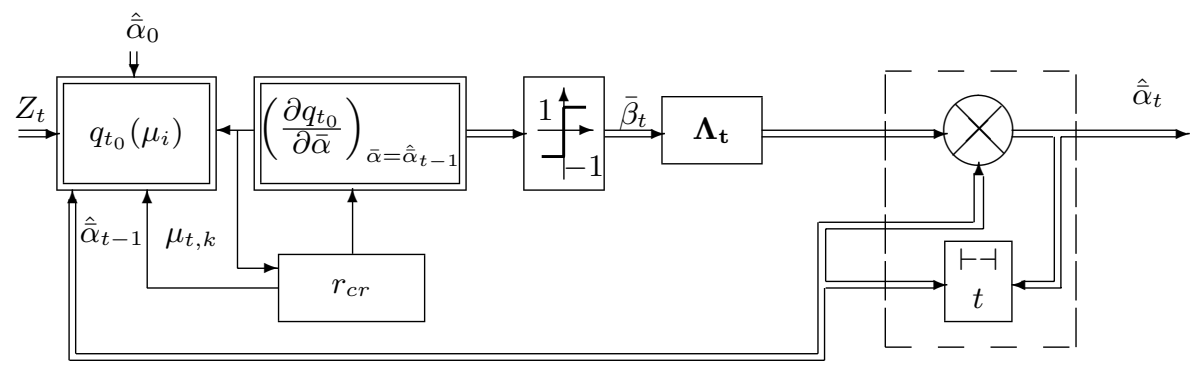

Fig. 1. The algorithm (7) structural chart

The structural chart of the described algorithm is shown on fig.1. It contains functional transformers realizing formation of $q_{t_{0}}\left(\mu_{t}\right)$ and $\left(\partial q_{t_{0}}\left(\mu_{t}\right) / \partial \bar{\alpha}\right)_{\bar{\alpha}=\hat{\alpha}_{t-1}}$, relay transformer with $\bar{\beta}_{i}\left(\mu_{t}\right)$ forming at its output, non-linear transformer $\boldsymbol{\Lambda}_{t}$ digrator and block $r_{c r}$ forming the value $\mu_{t, k}$ in accordance with (8). The value $\mu_{t, k}$ increases until the second of the conditions (7) is met and after that a permission for performance of an iteration enters the functional transformer $\left(\partial q_{t_{0}}\left(\mu_{t}\right) / \partial \bar{\alpha}\right)_{\bar{\alpha}=\hat{\alpha}_{t-1}}$.

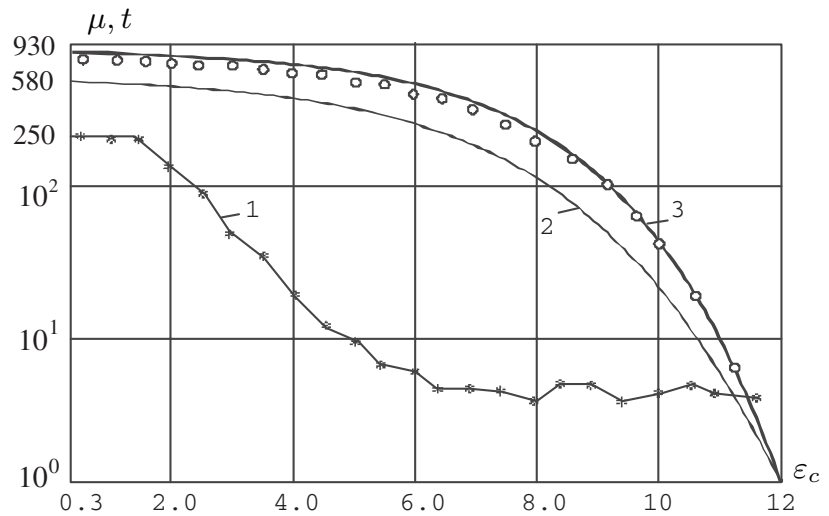

Fig. 2. The plot of connection between local sample size (number of iterations) and $\varepsilon_{c}\left(1\right.$ is $\mu$-plotted against $\varepsilon_{c} ; 2$ and 3 are $t$-plotted against $\varepsilon_{c}$ accordingly at $\mu=v a r$ and $\mu=$ const $=250 ; \diamond$ is experimental results at $\mu=$ var $)$ 
As an example let us present the results obtained when solving the problem of fragment position search on real images of the optical range (with correlation interval approximately equal to 5 steps of sample grid). On fig.2 the dependence of GF local sample size $\mu$ on the unbalance $\varepsilon_{c}$ of a fragment centre true position averaged on 800 realizations and its estimations under contrast of fragment scale to a reference image by $25 \%$, slewing angle, slewing angle $\varphi=30^{\circ}$ and initial unbalance $\varepsilon_{c}=12$ (curve 1 , experimental data is asterisks). It is clearly seen that $\mu$ increases only at relatively small $\varepsilon_{c}$. On the same figure dependence of iteration number $t$ on $\varepsilon_{c}$ is shown. The circles correspond to mean iteration numbers averaged on 800 realizations, curve 3 represents the mathematical expectation of iteration number calculated on the basis of the strategy [10]. It is seen that there is good correspondence between experimental and analytical results (circles and curve 3). In this case Gaussian image model with Gaussian correlation function $(\mathrm{CF})$ with signal variance to noise variance ratio equal to 0.01 was used. The algorithm parameters and initial approximations of the parameters vector are consistent with the experimental ones.

Curve 2 is consistent with the iteration number mathematical expectation for the situation of $\mu=$ const $=250$. The algorithm with $\mu=250$ obtains $M\left\{\varepsilon_{c}\right\}=0.3$ in 580 iterations. The algorithm with varying $\mu$ affords the same quality of estimation at 930 iterations (curve 2), but computer time expenses prove to be 9,6 times less.

\section{Conclusion}

The algorithms of multidimensional images sequences spatial deformations parameters estimation developed on the basis of pseudogradient non-identification procedures have high accuracy of estimates to be formed and fast speed of calculation and can be recommended for application in real-time systems. At absence of image brightness distortions it is expedient to choose interframe difference mean square as a goal function and at interframe brightness distortions close to linear-interframe correlation sample coefficient. When calculating the pseudogradient operations number reduction can be obtained by using goal function gradient contraction and substitution of deformed frame value prediction with a simpler estimate through interpolation. Regulation of goal function local sample volume during the algorithm processing enables to essentially reduce computational expenses. In the considered algorithm interframe correlation sample coefficient was used as a goal function. However the presented principles of development of pseudogradient algorithms with regulated volume of local sample can be also applied at other goal functions, in particular, mean square of interframe difference.

\section{References}

1. Tzypkin, Y. Z.: Information theory of Identification. Nauka: Fizmatlit, Moskow (1995) [in Russian] 
2. Tachlinskii, A.G.: Estimation of Image Distortions in Sequence of Frames. Pattern Recognition and Image Analysis, Vol.6(4) (1996) 728-732

3. Tachlinskii, A.G.: Image Sequence Spartial Deformation Parameters Estimation. UlGTU, Ulyanovsk (2000)[in Russian]

4. Tashlinskii, A.G.: Pseudogradient estimation of image sequence spatial deformations. Automation, Control and Inrormation Technology: A Publication of The International Association of Science and Technology for Development - IASTED. ACTA Press, Anaheim Calgary Zurich (2002) 382-385

5. Tashlinskii, A.G.: Pseudogradient estimation of image sequence spatial deformations. Naukoemkie Tehnologii, Vol.3(3) (2002) 32-43 [in Russian]

6. Polyak, B.T., Tsypkin, Ya.Z.: Pseudogradient Algorithms of Adaptation and Learning. Avtomatika i telemehanika, 6 (1973) 45-68 [in Russian]

7. Polyak, B.T., Tsypkin, Ya.Z.: Criterion Algorithms of Stochastic Optimization. Avtomatika i telemehanika, 6 (1984) 95-104 [in Russian]

8. Tachlinskii, A.G.: Estimation of Geometric Image Distortions in a Sequence of Frames. Pattern Recognition and Image Analysis, Vol.8(2) (1998) 258-259

9. Tachlinskii, A.G.: Pseudogradient Algorithms for Estimation Spatial Distortions of Image with Varying Volume of the Local Sample of the Goal Function. Pattern Recognition and Image Analysis, Vol.11(1) (2001) 247-250

10. Tachlinskii, A.G., Tikhonov V.O.: A method of multidimentional process parameters pseudogradient measurement error analysis. Izvestija vuzov: Radioelektronika, Vol.44(9) (2001) 75-80 [in Russian] 